\title{
Cooperative Optimal Control of Battery Energy Storage System under Wind Uncertainties in a Microgrid
}

DOI:

10.1109/TPWRS.2017.2741672

\section{Document Version}

Accepted author manuscript

Link to publication record in Manchester Research Explorer

\section{Citation for published version (APA):}

Zhao, T., \& Ding, Z. (2017). Cooperative Optimal Control of Battery Energy Storage System under Wind Uncertainties in a Microgrid. IEEE Transactions on Power Systems, PP(99).

https://doi.org/10.1109/TPWRS.2017.2741672

\section{Published in:}

IEEE Transactions on Power Systems

\section{Citing this paper}

Please note that where the full-text provided on Manchester Research Explorer is the Author Accepted Manuscript or Proof version this may differ from the final Published version. If citing, it is advised that you check and use the publisher's definitive version.

\section{General rights}

Copyright and moral rights for the publications made accessible in the Research Explorer are retained by the authors and/or other copyright owners and it is a condition of accessing publications that users recognise and abide by the legal requirements associated with these rights.

\section{Takedown policy}

If you believe that this document breaches copyright please refer to the University of Manchester's Takedown Procedures [http://man.ac.uk/04Y6Bo] or contact uml.scholarlycommunications@manchester.ac.uk providing relevant details, so we can investigate your claim.

\section{OPEN ACCESS}




\title{
Cooperative Optimal Control of Battery Energy Storage System under Wind Uncertainties in a Microgrid
}

\author{
Tianqiao Zhao, Zhengtao Ding, Senior Member, IEEE,
}

\begin{abstract}
Since high penetration renewable sources are integrated into the future power system, energy storage systems are often installed to maintain the frequency stability in a microgrid. The operation mode of a microgrid may frequently change due to the intermittency of renewable sources, and energy storage systems will be charged/discharged accordingly to smooth and balance the generation of renewable sources. Thus, energy storage systems should be coordinated in a proper approach to ensure the supply-demand balance while increasing their profits and energy efficiency. To this end, a distributed optimal solution for energy storage systems to maintain the supplydemand balance while maximizing their welfare and energy efficiency is proposed to energy storage systems by enhancing the coordination through the communication under a multiagent system framework. Under this framework, each energy storage system is designated as an agent, and it only utilizes the local information to interact with the neighbouring agents. Additionally, since the participants in microgrid may not be willing to release their information about cost functions, or even the local gradient with other neighbouring agents, the proposed solution could be implemented without these private information to the individual agents. The simulation studies are carried out for IEEE 14-bus and 30-BESS systems to validate the effectiveness of the proposed distributed solution.
\end{abstract}

Index Terms-energy storage systems, distributed cooperative control, microgrid, multi-agent system, wind uncertainties

\section{INTRODUCTION}

$\mathrm{T}^{\mathrm{H}}$ HE microgrid is a promising solution to integrate controllable power electronics devices and advanced management and protection technology into the electricity network [1]. Since the emerging technologies, such as renewable energy sources and battery energy storage systems (BESSs), grow rapidly to make the grid eco-friendly, these technologies are expected to undertake the responsibility of the system stability.

With a proper designed pitch angle and rotor speed control strategy [2], [3], the effectiveness of the wind power generation for maintaining the grid frequency stability has been verified by several existing researches in different areas such as inertial, primary and second frequency control [4], [5]. However, due to the intermittency of wind power generation supply, new challenges are posed to the operation and control of a microgrid, especially under high penetration levels. As a result, the stability of the microgrid would be affected by integrating high penetration wind power generation, which may cause a mismatch between supply and demand when the available wind power generation is not equal to total load demand.
To overcome this problem, a solution is to install BESSs for the intermittent renewable sources [6]-[8], since they can provide a faster response to absorb excessive power and compensate the insufficient power during peak generation and load periods, respectively. Thus, the active power imbalance caused by integrating high penetration wind power generation can be addressed by introducing and coordinating the BESSs in a well-designed cooperative manner.

The cooperative approaches in the existing studies are mainly clarified into three categories, namely centralized schemes, decentralized approaches and distributed control strategies. The centralized schemes work well for conventional power system by introducing a microgrid central controller [9], [10]. The methods implemented in a centralized manner require an information centre to collect global information and a central controller to process the amounts of received data [11]. Thus, the required computational capacity of the control centre is rapidly growing with the increase of the grid scale and it may be more vulnerable to the single-point failures. The decentralized approaches may be robust and less costs in terms of no communication network is needed. However, in [12], it shows that the available resources in the network would be not utilized in a cost-effective way because of the deficiency of broader available information. In contrast, the cooperative solution in the distributed way that only utilizes local information through a local private communication network [13]. In light of the smart grid, it will consist of more distributed controllable power-electronics devices with the ability to exchange information through a communication network. Therefore, the emerging management solution should be efficient and low-cost for an economically viable microgrid.

Recently, the control and optimization of BESSs have drawn the attention of researchers [14]-[17]. In [14], the size of operation BESSs is optimized based on adjusting state of charge (SoC) limits. In [15], group BESSs are coordinated by a distributed control algorithm for voltage and frequency deviation regulation. To achieve the SoC equalization, authors in [16] improved the conventional droop control by modifying a virtual droop resistance according to the SoC imbalance. A cooperative control method is presented in [17] for BESSs based on time-of-use (ToU) pricing. However, the relevant results in the existing literatures are designed by assuming the energy efficiency of multiple BESSs remains as a constant value. It is indicated that the variation of charging/discharging efficiency of multiple BESSs is indispensable [18]. Addition- 
ally, the experiment in [19] shows that the energy efficiency fluctuates drastically according to the charging/discharging rate and SoC. In this case, the energy efficiency should be taken into consideration in the optimization and control design of multiple BESSs.

In this paper, a novel distributed algorithm is proposed to coordinate multiple BESSs under wind uncertainties by maximizing the total welfare of BESSs while respecting the supplydemand balance. By considering the energy efficiency and ToU pricing, an objective function is formulated to maximize the total welfare of multiple BESSs that can encourage BESSs to participate in grid regulation. Furthermore, a coordination scheme of BESSs under wind power generation uncertainties is proposed for multiple BESSs to maintain active power balance. To make the proposed algorithm more compatible with the requirement of the power grid, the multi-agent system (MAS) framework is further developed in this paper. By regarding each BESS as an agent, it only needs to exchange information with its neighbouring agents through a local communication network. Thus, the proposed strategy can work in a distributed way so that computational and communication burdens are reduced comparing with centralized methods. Besides, the information sharing of the cost function may result in the privacy concerns of the participants in the microgrid. To this end, in this paper, the proposed algorithm solves the formulated problem privately which is achieved by introducing a mismatch estimator to update the local power output and removing the requirement of gradient information sharing. A comparison study is first introduced to present the advantage of the proposed algorithm. Then, the effectiveness and the scalability of our algorithm are further validated through simulation studies under different operation conditions of simulation studies in IEEE 14-bus system and a 30-BESS system, respectively.

The remainder part of the paper is organized as follows. Section II outlines the multiple BESS system architecture and gives a description of the network model under a microgrid environment. Section III introduces the distributed energy management of BESSs. Section IV proposes a distributed optimal solution for the energy management problem of multiple BESSs. Section V presents the simulation results and corresponding analysis. Finally, the conclusion is drawn in Section VI.

\section{Network Model of Multiple Battery EnERgy StORAGE Systems}

\section{A. Overview of Multiple Battery Energy Storage Systems}

Fig. 1 presents a MAS framework for an architecture of multiple BESSs and wind power generations, which is connected to the main grid through the Point of Common Coupling (PCC). The PCC of the main grid is used to observe the power delivered/withdrawn and decide the operation mode of the microgrid. Each BESS consists of several lithium-ion batteries interfaced with the DC-AC inverter. In the proposed framework, a BESS is designated as an agent, and controlled by an energy management system (EMS). A communication network is embedded in the EMS to transfer the information

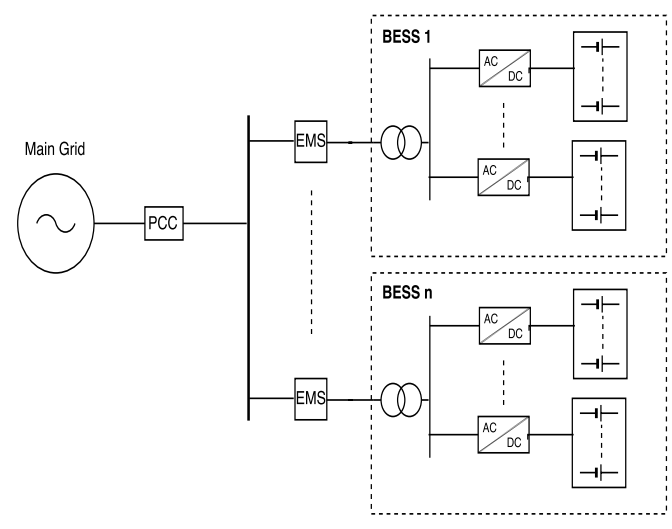

Fig. 1. Multiple BESSs System

with its neighbouring agents that to achieve a determined objective. In addition, following the control strategy in [20], a storage system controller (SSC) is applied to adjust the power output of the BESS to the signal generated by the communication network.

\section{B. Network Model Based on the Multi-agent Framework}

The proposed MAS framework consists of $\mathcal{N}$ distributed BESSs, which are assigned as controllable agents. A weightedbalanced directed graph $\mathcal{G}=(\mathcal{V}, \mathcal{E})$ is constructed to represent the communication topology of the network, where $\mathcal{V}=\left\{\nu_{1}, \ldots, \nu_{n}\right\}$ denotes the agent set and $\mathcal{E}$ is the edge set. Assuming that there exists a directed path that connects any pair of BESSs, and the weight of an edge from $i$ th agent to $j$ th agent is $a_{i j}>0$, and the weight on the self loop of $i$ th agent is $a_{i i}=0$, respectively. In the communication network, each BESS has ingoing and outgoing edges, and the weighted in-degree and out-degree of $i$ th agent are $d_{i n}(i)=\sum_{j=1}^{n} a_{i j}$ and $d_{\text {out }}(i)=\sum_{j=1}^{n} a_{j i}$, respectively. It should be noted that $d_{\text {out }}(\nu)=d_{\text {in }}(\nu)$ for all agents because the communication network is weighted-balanced. The Laplacian matrix $\mathcal{L}$ associated with $\mathcal{G}$ is defined as $\mathcal{L}_{i i}=\sum_{j \neq i} a_{i j}$ and $\mathcal{L}_{i j}=-a_{i j}$, $i \neq j$. As indicated in [20], the designed communication network is independent of the power network, and hence it can be constructed in a cost-effective approach based on practical requirements of a microgrid.

\section{Distributed ENERGY MANAGEMENT OF BATTERY ENERGY STORAGE SYSTEMS UNDER WIND POWER UNCERTAINTIES}

In a microgrid, the frequency may change rapidly and frequently due to the uncertainties of wind power generation, which is mainly influenced by the supply-demand mismatch of the active power. The active power balance at $t_{0}$ in a microgrid can be represented as

$$
\sum_{k \in S_{W}} P_{W, k}^{0}+\sum_{i \in S_{B}} P_{B, i}^{0}=\sum_{j \in S_{l}} P_{l, j}^{0},
$$

where $S_{W}, S_{B}$ and $S_{l}$ are the index set of wind turbines (WTs), BESSs and load demands, respectively; $P_{B, i}^{0}$ is the 
charging/discharging power of $i$ th BESS that can be positive/negative; $P_{W, k}^{0}$ and $P_{l, j}^{0}$ are the wind power generation and load demand of $k$ th WT and $j$ th load, respectively.

Although wind power generation can relieve the fatigue of the frequency regulation, it is deficient in terms of accuracy due to its intermittent nature. Furthermore, when wind power generations are controlled in the MPPT mode, it cannot be treated as dispatchable. In contrast, BESSs have the fast response property and exhibit high performance. As a result, installing BESSs in a power grid is a promising solution for absorbing excessive power and compensating the insufficient power.

Then the net power required from the BESS for the frequency regulation in a short-term can be calculated as

$$
\sum_{i \in S_{B}} P_{B, i}=\Delta P_{D}=\sum_{k \in S_{W}} \Delta P_{W, k}-\sum_{j \in S_{l}} \Delta P_{l, j},
$$

where $P_{B, i}$ is the power output of $i$ th BESS; $\Delta P_{W, k}$ and $\Delta P_{l, j}$ are active power change of $k$ th WT and $j$ th load, respectively.

However, as indicated in [19], the output power level is one of the factors affecting the energy efficiency of a BESS. Thus, a factor called inner energy rate is defined as (3) to express the rate of inner charging or discharging energy of a BESS for output power.

$$
\varepsilon_{i}=\frac{\Delta E_{B, i}}{P_{B, i}} .
$$

where $\Delta E_{B, i}$ is the change of battery's inner energy. Then the actual output power is calculated as

$$
P_{B, i}^{o}=\varepsilon_{i} P_{B, i} \text {. }
$$

Note that the electricity price is an important factor that can encourage the BESS to participate the frequency regulation. As indicated in [21]-[23], the electricity price is an effective method of energy management for the participants in a microgrid to adjust their electricity consumption behaviors in a cost-effective way. In this paper, we adjust the ToU pricing, $\rho(t)$, as a variable to coordinate BESSs to discover the maximum efficient point of the operation. Thus, the objective is defined as maximizing the total welfare of BESSs by adjusting the price while considering the efficiency of the BESSs and maintaining the active power balance, such as

$$
\operatorname{Max} \sum_{i \in S_{B}}\left(\rho(t) P_{B, i}-\varepsilon_{i} P_{B, i}\right)
$$

where $\varepsilon_{i}$ is the inner rate energy. The experiment in [19] shown that the function of the inner rate energy can be written in a piecewise linear function as

$$
\varepsilon_{i}=\alpha_{i} P_{B, i}+\beta_{i} .
$$

By substituting (6) into (5), the objective function can be rewritten as

$$
\begin{array}{cc}
\operatorname{Max} \quad \sum_{i \in S_{B}}\left(\rho(t) P_{B, i}-\left(\alpha_{i} P_{B, i}^{2}+\beta_{i} P_{B, i}\right)\right) \\
\text { s.t } & \sum_{i \in S_{B}} P_{B, i}=\Delta P_{D} \\
& P_{B, i}^{m} \leq P_{B, i} \leq P_{B, i}^{M}
\end{array}
$$

where $P_{B, i}^{m}$ and $P_{B, i}^{M}$ are the lower and upper bound of $i$ th BESS, respectively. The equality constraint describes the balance between the net power demand and the total output power of BESSs, and the inequality constraint are the local boundary of the output power for $i$ th BESS.

It should be noted that the transmission losses are inevitable, and these are about $5 \%-7 \%$ of the total load according to the energy information administration (EIA) [24]. Thus, it can be modelled by multiplying the load by $5 \%-7 \%$.

For multiple BESSs, they may be operated on different $\mathrm{SoC}$ due to the efficiency difference. Since the power is predominately shared among BESSs with higher SoC, some BESS will be overloaded even the required power is lower than the total power capacities of BESSs. As a result, a proper power sharing method is required to coordinate BESSs based on their energy level. To this end, the objective function is rewritten as,

$$
\begin{array}{cc}
\text { Min } & \sum_{i \in S_{B}} f_{i}\left(P_{B, i}\right) \\
\text { s.t } & \sum_{i \in S_{B}} \omega_{i} P_{B, i}=\Delta P_{D} \\
& h_{i}\left(P_{B, i}\right)=\left(P_{B, i}^{m}-P_{B, i}\right)\left(P_{B, i}^{M}-P_{B, i}\right) \leq 0,
\end{array}
$$

where $f_{i}\left(P_{B, i}\right)=\left(\alpha_{i} P_{B, i}^{2}+\beta_{i} P_{B, i}\right)-\rho(t) P_{B, i} \cdot \omega_{i}$ is the weight on the contribution of $i$ th BESS. It can be defined as a ratio of the energy level in order to prevent BESSs from running out prematurely, i.e.,

$$
\omega_{i}=\frac{E_{i}}{\sum_{i \in S_{B}} E_{i}}
$$

where $E_{i}$ is the energy level of $i$ th BESS.

A central coordinator could be deployed to solve the above problem. The coordinator communicates with each BESS in the network by a bi-directional communication line to collect the data required to solve the problem, such as the objective functions, the operation constraints and the actual power output. With the revived data, it solves the problem and broadcasts the reference to all connected BESSs. However, due to the high penetration of wind power generations, the centralized strategy may lose its control efficiency if operating conditions change frequently and unpredictably. In the following section, a distributed algorithm is proposed to coordinate the BESSs to maintain the active power balance, and in the meantime, a coordination scheme of BESSs and WTs is presented to overcome the problem of wind power generation errors.

Remark 3.1: A model of the distribution loss can be adopted to estimate the value of these loss according to [25] ([25] in the manuscript). Then, the total demand required from BESSs can be recalculated including these available values. As a result, the network can discover a new optimization result by considering the distribution loss.

\section{Consensus-based Cooperative Algorithm DESIGN}

The formulated optimization problem of BESSs in (8) is a convex problem with both equality and inequality con- 
straints. In this section, the solution set is firstly characterized by the so-called refined Slater condition and the Karush-Kuhn-Tucker (KKT) optimality conditions. A consensus-based distributed cooperative algorithm is presented for discovering the optimal solution of the energy management problem. Then the implementation of the proposed algorithm is presented.

\section{A. Solution Set of Distributed Energy Management}

Let $L$ be the Lagrangian equation with the Lagrangian multiplier $\lambda$, one has

$$
\begin{aligned}
L & =\sum_{i \in S_{B}}\left(\left(\alpha_{i} P_{B, i}^{2}+\beta_{i} P_{B, i}\right)-\rho(t) P_{B, i}\right) \\
& +\left(\sum_{i \in S_{B}} \lambda_{i} \omega_{i} P_{B, i}-\Delta P_{D}\right),
\end{aligned}
$$

where $\lambda_{i}$ is the Lagrange multiplier and let $\lambda=\left\{\lambda_{1}, \ldots, \lambda_{n}\right\}$. It should be noted that the inequality constraints are unnecessary to be added in the augmented function due to they are local and can be treated as boundaries of the problem domain [26]. These inequality constraints can be taken into account by applying additional projection operations, which do not affect the convergence analysis as shown in [27].

Remark 4.1: By using the dual decomposition [28], one has

$$
\begin{gathered}
L=\sum_{i \in S_{B}}\left(\left(\alpha_{i} P_{B, i}^{2}+\beta_{i} P_{B, i}\right)\right. \\
\left.-\left(\rho(t)-\lambda_{i} \omega_{i}\right) P_{B, i}\right)+\Delta P_{D} .
\end{gathered}
$$

It can be found that the Lagrange multiplier $\lambda_{i}$ acts as a virtual price to adjust the real price so that BESSs are coordinated to find the maximum efficient point.

Since the energy management problem is a convex optimization problem with affine constraints, the global optimality can be ensured by using KKT optimality conditions [29]. Following the KKT conditions, a point $P_{B}^{*}=\left\{P_{B, 1}^{*}, \ldots, P_{B, n}^{*}\right\} \in$ $\mathcal{R}^{n}$ is a solution of the energy management problem if and only if there exists a point $\lambda^{*}$ such that

$$
\begin{gathered}
\nabla f_{i}\left(P_{B, i}^{*}\right)+\lambda^{*} \omega_{i}=0, \quad i \in\{1, \ldots, n\} \\
\omega_{1} P_{B, 1}^{*}+\cdots+\omega_{i} P_{B, i}^{*}=\Delta P_{D} .
\end{gathered}
$$

\section{B. Distributed Cooperative Algorithm Design}

In this section, a distributed algorithm is presented for the energy management of BESSs. Following [30], the active power dynamics of $i$ th BESS can be written as

$$
\dot{P}_{B, i}=u_{B, i},
$$

where $u_{B, i}$ is the control input for $i$ th BESS. In a microgrid, the distributed energy management of BESSs aims to maximize the efficiency of BEESs under a proper designed strategy, which can be achieved by controlling the references of BESSs. Then the design objective is defined such that the control input only requires the information from neighbouring BESSs, and then $P_{B, i}$ converges to the solution set of the proposed problem (8).
To this end, the solution for the problem (8) is the following continuous-time distributed algorithm,

$$
\begin{aligned}
& \dot{P_{B, i}}=-\nabla f_{i}\left(P_{B, i}\right)-\omega_{i} \lambda_{i} \\
& \dot{\lambda_{i}}=-\gamma_{1} \sum_{i \in n} a_{i j}\left(\lambda_{i}-\lambda_{j}\right)-\gamma_{2} \sum_{i \in n} a_{i j}\left(z_{i}-z_{j}\right) \\
& \quad+\gamma_{3}\left(\omega_{i} P_{B, i}-r_{i} \Delta P_{D}\right) \\
& \dot{z_{i}}=\sum_{i \in n} a_{i j}\left(\lambda_{i}-\lambda_{j}\right)
\end{aligned}
$$

where $\gamma_{1}, \gamma_{2}$ and $\gamma_{3}>0$ are the positive constants, respectively. We assume $r_{i}$ is the ability that $i$ th BESS is able to detect the total required load, and denote $r=\left[r_{1}, \ldots, r_{n}\right]^{T}$ with $\mathbf{1}_{n}^{T} r=1$, for $i \in n$., where $\mathbf{1}_{n}=[1,1, \ldots]^{T} \in \mathbb{R}^{n}$.

To solve the optimization problem in a distributed way, inspired by the centralized saddle-point dynamics in [31], a mismatch estimator is introduced by taking advantage of the idea of distributed average estimation to observe the global information. With the estimator to observe the supply-demand mismatch, the algorithm (15) then can be implemented in a distributed way since the only information required is the states of $i$ th BESS, i.e. $\lambda_{i}$ and $z_{i}$. As a result, each BESS only needs to send/receive the value of $\lambda_{i}$ and $z_{i}$ to its neighbour BESSs. With considering the privacy concerns of BESSs, the proposed algorithm does not require BESSs to share their gradients of the cost function with neighbouring BESSs. Furthermore, in view of Remark. 4.1, the ToU signal $\rho(t)$ will be adjusted by $\lambda_{i}$ so that multiple BESSs are coordinated to discover the most efficient point.

According to (15), the control input for $i$ th BESS is designed as

$$
u_{B, i}=-\nabla f_{i}\left(P_{B, i}\right)-\omega_{i} \lambda_{i} .
$$

Remark 4.2: To keep the BESS operating in a feasible mode, various local constraints can be integrated in the proposed algorithm, such as the SoC constraint, the constraints of minimum/maximum charging/discharging duration. These local constraints can be dealt with by the proposed algorithm by including corresponding projection operation.

\section{Convergence Analysis}

To facilitate convergence analysis, the proposed algorithm (15) is written in a compacted form, such as

$$
\begin{aligned}
& \dot{P_{B}}=-\nabla f\left(P_{B}\right)-\omega \lambda \\
& \dot{\lambda}=-\gamma_{1} \mathcal{L} \lambda-\gamma_{2} \mathcal{L} z+\gamma_{3}\left(\omega P_{B}-r \Delta P_{D}\right) \\
& \dot{z}=\mathcal{L} \lambda
\end{aligned}
$$

where $P_{B}=\left[P_{B, 1}, \ldots, P_{B, n}\right]^{T}, \lambda=\left[\lambda_{1}, \ldots, \lambda_{n}\right]^{T}$, $\nabla f\left(P_{B}\right)=\sum_{i \in S_{B}} f_{i}\left(P_{B, i}\right)$ and $z=\left[z_{1}, \ldots, z_{n}\right]^{T}$, respectively, and let $\omega=\operatorname{Diag}\left(\omega_{1}, \ldots, \omega_{n}\right)$. The inspiration of our algorithm is based on multiple time-scale operations, which applies a distributed estimator to distribute the centralized saddle-point algorithm. To analyze the convergence, we first consider the equilibrium point $\left(\bar{P}_{B}, \bar{\lambda}, \bar{z}\right)$ of the proposed algorithm. When executing the algorithm over a connected and weight-balanced graph, it results in

$$
\mathbf{1}_{n}^{T} \dot{z}=\mathbf{1}_{n}^{T} \mathcal{L} \bar{\lambda}=0,
$$


where the fact that $\mathbf{1}_{n}^{T} \mathcal{L}=0$ is used to deduce (18). Then, the equilibrium point can be obtained by

$$
\begin{aligned}
& 0=-\nabla f\left(\bar{P}_{B}\right)-\omega \bar{\lambda} \\
& 0=-\gamma_{1} \mathcal{L} \bar{\lambda}-\gamma_{2} \mathcal{L} \bar{z}+\gamma_{3}\left(\omega \bar{P}_{B}-r \Delta P_{D}\right) \\
& 0=\mathcal{L} \bar{\lambda}
\end{aligned}
$$

Left multiplying (19c) by $\mathbf{1}_{n}^{T}$ gives

$$
\begin{aligned}
& \gamma_{3} \mathbf{1}_{n}^{T}\left(\omega \bar{P}_{B}-r P_{D}\right)=0 \\
& \nabla f\left(\bar{P}_{B}\right)+\omega \bar{\lambda} \mathbf{1}_{n}=0 .
\end{aligned}
$$

Thus, it is shown that the equilibrium point $\left(\bar{P}_{B}, \bar{\lambda}, \bar{z}\right)$ satisfies

$$
\bar{P}_{B, i}=P_{B}^{*}, \quad \bar{\lambda}_{i}=\lambda^{*}, \quad \bar{z}_{i}=z^{*}, \text { for } i \in n,
$$

where $\left(P_{B}^{*}, \lambda^{*}, z^{*}\right)$ is the solution set of the optimization problem given in (13). For the convenience of the convergence analysis, the states in (17) are translated to the equilibrium point as

$$
\hat{P}_{B}=\left(P_{B}-\bar{P}_{B}\right), \quad \hat{\lambda}=m^{T}(\lambda-\bar{\lambda}), \quad \hat{z}=m^{T}(z-\bar{z}),
$$

where $\left(\bar{P}_{B}, \bar{\lambda}, \bar{z}\right)$ is any equilibrium point of (17) and $m$ can be defined as in [32]. Thus, one has

$$
\begin{aligned}
& \dot{\hat{P}}_{B}=-\phi-\omega m \hat{\lambda} \\
& \dot{\hat{\lambda}}=-\gamma_{1} m^{T} \mathcal{L} m \hat{\lambda}-\gamma_{2} m^{T} \mathcal{L} m \hat{z}-\gamma_{3} \omega m \hat{P}_{B} \\
& \dot{\hat{z}}=m^{T} \mathcal{L} m \hat{\lambda}
\end{aligned}
$$

where $\phi=\left(\nabla f\left(\hat{P}_{B}+\bar{P}_{B}\right)-f\left(\bar{P}_{B}\right)\right)$. To study the stability of the proposed algorithm, we consider a candidate Lyapunov function

$$
V=\frac{1}{2} \gamma_{3} \hat{P}_{B}^{T} \hat{P}_{B}+\frac{1}{2} \hat{\lambda}^{T} \hat{\lambda}+\frac{1}{2} \gamma_{2} \hat{z}^{T} \hat{z} .
$$

The Lie derivative of $V$ along with (23) is given as

$$
\begin{aligned}
\dot{V} & =\gamma_{3} \hat{P}_{B}^{T}(-\phi-\omega m \hat{\lambda})+\gamma_{2} \hat{z}^{T}\left(m^{T} \mathcal{L} m \hat{\lambda}\right) \\
& +\hat{\lambda}^{T}\left(-\gamma_{1} m^{T} \mathcal{L} m \hat{\lambda}-\gamma_{2} m^{T} \mathcal{L} m \hat{z}-\gamma_{3} \omega m \hat{P}_{B}\right) \\
& =-\gamma_{3} \hat{P}_{B}^{T} \phi-\gamma_{1} \hat{\lambda}^{T} m^{T} \mathcal{L} m \hat{\lambda} \\
& \leq-\gamma_{3}\left(\hat{P}_{B}+P_{B}^{*}-P_{B}^{*}\right) \phi-\gamma_{1} \hat{\lambda}^{T} m^{T} \mathcal{L} m \hat{\lambda}
\end{aligned}
$$

where the convexity of the cost function, i.e., $\gamma_{3}\left(\hat{P}_{B}+P_{B}^{*}-\right.$ $\left.P_{B}^{*}\right) \phi \geq 0$ is invoked for obtaining the last inequality. So far, it is shown that the trajectories of (23) and also (15) are bounded as $\dot{V} \leq 0$. With the invariant set Theorem 1 in [32], it can be concluded that the points on $\dot{V}=0$ are the equilibrium points of the algorithm.

\section{The Coordination of BESSs under Wind Power Generation Error}

Due to the intermittent nature of the wind power generation, it may lose its accuracy for maintaining supplydemand balance, whereas BESSs have the characteristic of the fast response and high performance for balancing shortterm variations of the network power. For this reason, the wind power generations are supplemented by the BESSs in real application.

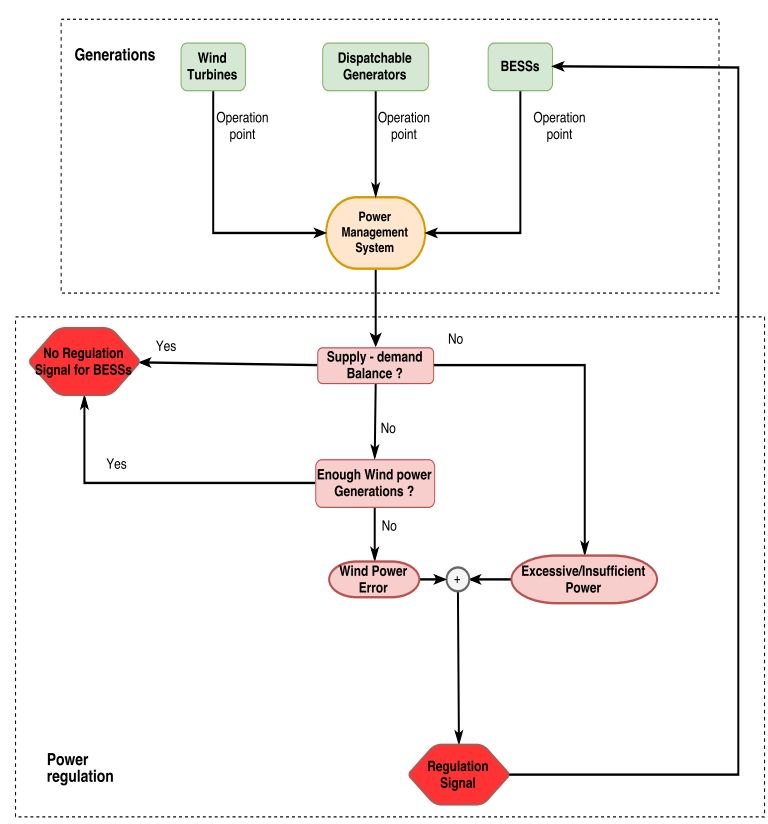

Fig. 2. Coordination scheme of wind generations and BESSs

As depicted in Fig. 2, a brief scheme is provided to coordinate the BESSs and wind power generations for maintaining the system stability. The power management system (PMS) can collect the operation points of all participants in the grid to observe the supply-demand balance. As shown in power regulation part, if there is unbalance in net power, wind power will be employed to compensate the mismatch as much as possible firstly. However, the wind power may be insufficient and inaccurate, and there are also control errors of the wind power generations. Therefore, a regulation signal will be generated based on both the mismatched power and the control error of wind power generations. Following the regulation signal $\left(\Delta P_{D}\right)$, BESSs are then implemented to absorb the excessive power or compensate the insufficient power according to the proposed algorithm.

\section{E. Algorithm Implementation}

With designating each BESS as an agent, the proposed algorithm is implemented in a distributed manner under a MAS framework. Each agent consists of two control levels. The top control level consists of three function modules, i.e., the measurement module, the communication module and the optimal solution discovery module. The measurement module obtains and updates the local information, and the communication module exchanges the information with the neighbouring agents. With the information provided by the measurement and communication module, the optimal solution discovery module updates the information and generates the output power reference for the bottom control level. Once the power reference is generated, the bottom-level control is implemented to control the agent to track this reference, which can follow the control scheme introduced in Fig. 3. 


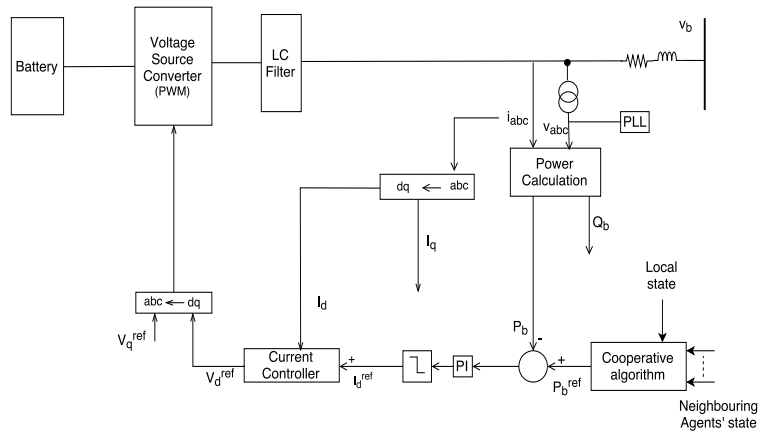

Fig. 3. The control diagram for a BESS

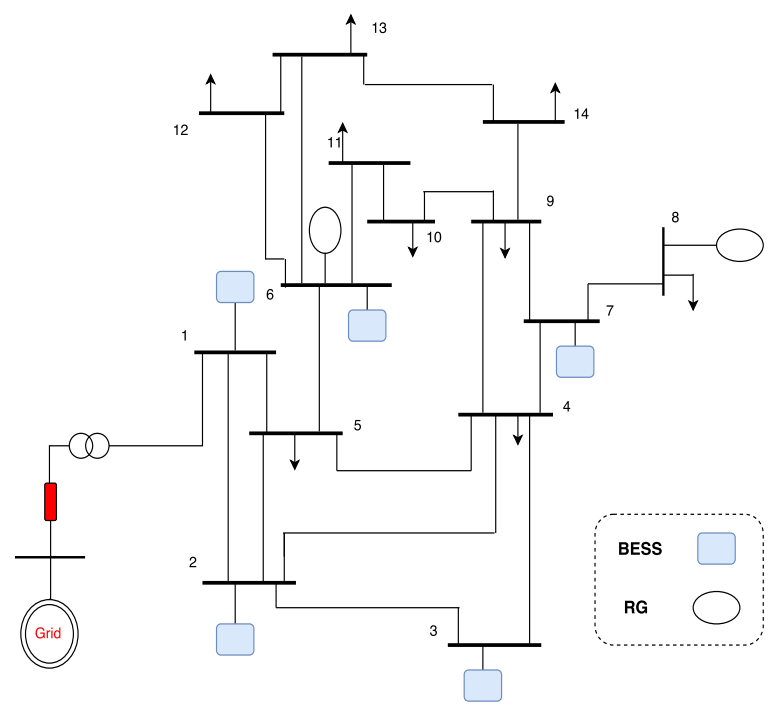

Fig. 4. IEEE 14-bus system with five BESSs

\section{Simulation Results AND ANALysis}

In this section, four case studies are provided to verify the effectiveness of the proposed optimization algorithm. In Case 5.1, the proposed algorithm is compared with another resource management approach to show the advantages of our algorithm. In Case 5.2, a modified IEEE 14-bus system with five BESSs and two WTs, as depicted in Fig. 4, is built in the MATLAB/Simulink that to demonstrate the convergence of the proposed algorithm under constant renewable generation and load demand. Case 5.3 is carried out with uncertain output power from the renewable generation. Finally, the scalability of the proposed algorithm is investigated in Case 5.4 where a 30-BESSs system is built in the MATLAB/Simulink.

During the simulation studies, the ToU signal is adopted as Fig. 7 to imitate the tariff in real application, which simplifies the signal in [33] but keeping the same property. The simulation parameters are summarised in Table. I that are adopted from the experimental results in [19], and the topology of the communication network is implemented to be identical to the physical network.

\section{A. Case 5.1}

To reveal the effectiveness of the proposed algorithm, our algorithm is first compared with another continuous algorithm
TABLE I

PARAMETERS OF SIMULATION STUDIES

\begin{tabular}{llllll}
\hline & $\alpha_{i}$ & $\beta_{i}$ & $P_{B, i}^{\min }(\mathrm{kW})$ & $P_{B, i}^{\max }(\mathrm{kW})$ & $\omega_{i}$ \\
\hline BESS1 & 0.2037 & 1.0460 & 0 & 60 & 0.20 \\
BESS2 & 0.2815 & 1.2309 & 0 & 65 & 0.16 \\
BESS3 & 0.1987 & 1.0292 & 0 & 68 & 0.24 \\
BESS4 & 0.2092 & 1.1245 & 0 & 62 & 0.22 \\
BESS5 & 0.2247 & 1.0996 & 0 & 70 & 0.18 \\
\hline
\end{tabular}

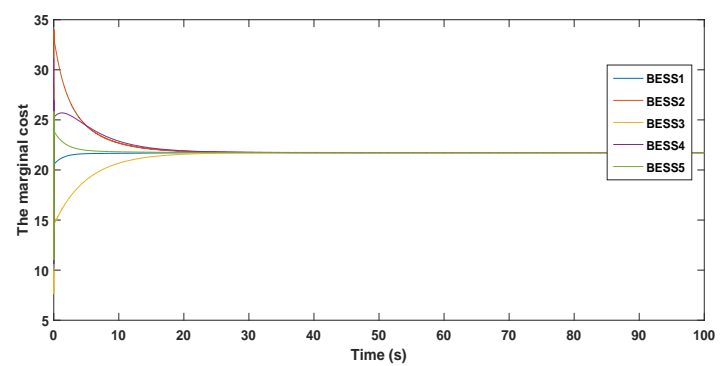

Fig. 5. The marginal cost update under the proposed algorithm

in [34]. Without loss of generality, the ToU pricing and the supply-demand mismatch are assumed to be a constant value and the operation condition is assumed to be same for this comparison study. As shown in Figs. 5 - 6, the marginal cost of each BESS can converge to its optimal value under both of the algorithms. However, by replacing the global mismatch estimator by a distributed estimator, the convergence speed of the proposed algorithm is faster than the algorithm in [34]. Additionally, the proposed algorithm guarantees the optimality without sharing the information about its own cost function.

\section{B. Case 5.2}

In this case study, the performance of the proposed distributed strategy is investigated in the IEEE 14-bus system. The microgrid is operated in the islanded mode and the supplydemand mismatch is set to be a constant value as $180 \mathrm{~kW}$. As shown in Fig. 8, the marginal cost of each BESS converges to the optimal value, and with the change of the ToU tariff during the simulation, the marginal cost will converge to a new optimal value according the price signal. Figs. 9 - 11 depict the output power references, the supply-demand mismatch estimation, and the total output power of BESSs, respectively. The results show that the output power references converge to the optimal value according to the marginal cost update and

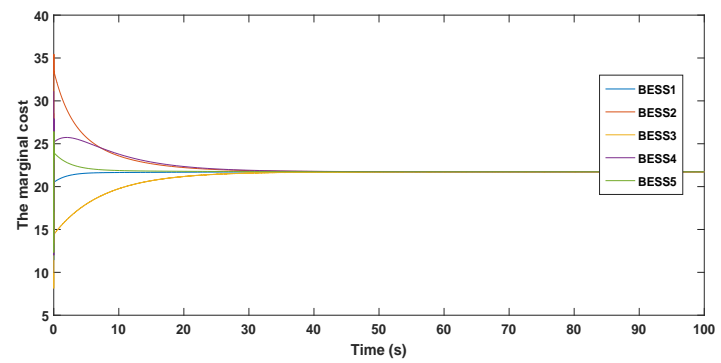

Fig. 6. The marginal cost update under the algorithm in [34] 


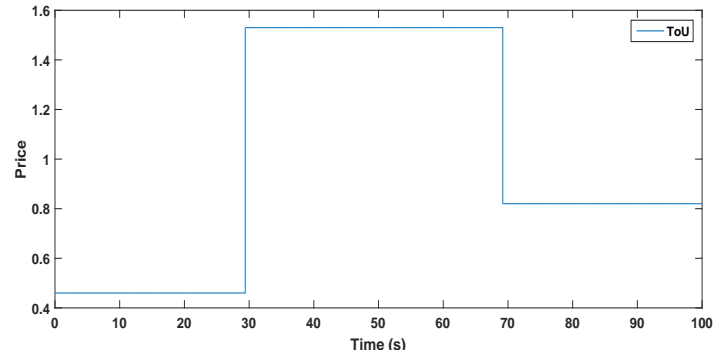

Fig. 7. The time of use tariff

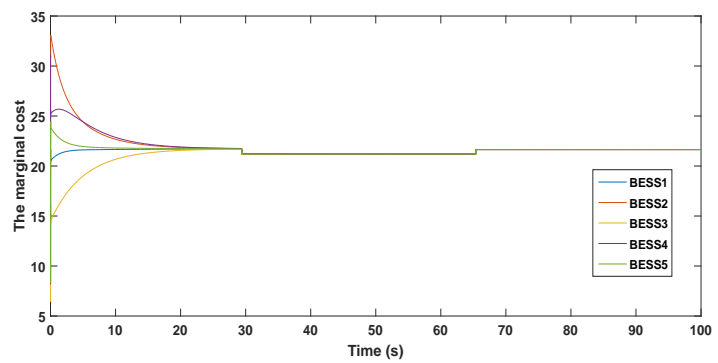

Fig. 8. The marginal cost update under the proposed algorithm

meanwhile, the supply-demand mismatch is eliminated by the proposed algorithm, which indicates its promising application in real-time control.

\section{Case 5.3}

Since the output power from the wind power generations will be uncertain and time-varying, the required power from BESSs will be time-varying when high penetration renewable sources are integrated into the microgrid. In this case, a simulation study is carried out under a time-varying output power from the wind power generation to verify the effectiveness

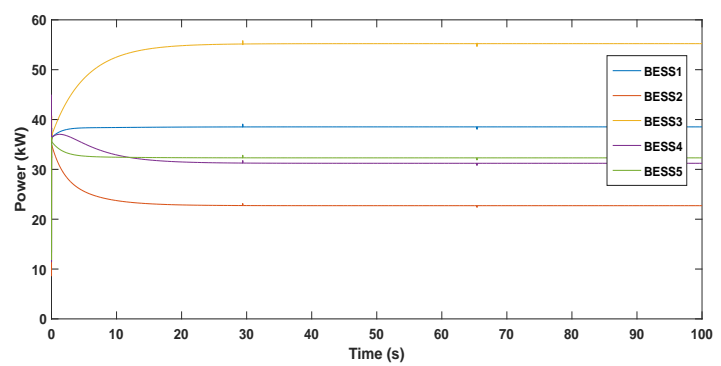

Fig. 9. The output power update under the proposed algorithm

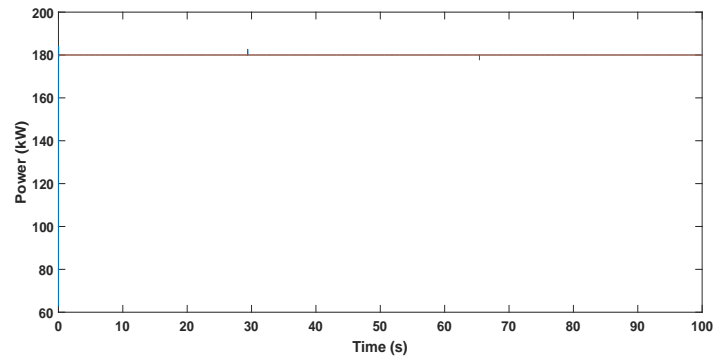

Fig. 10. The power balance estimation

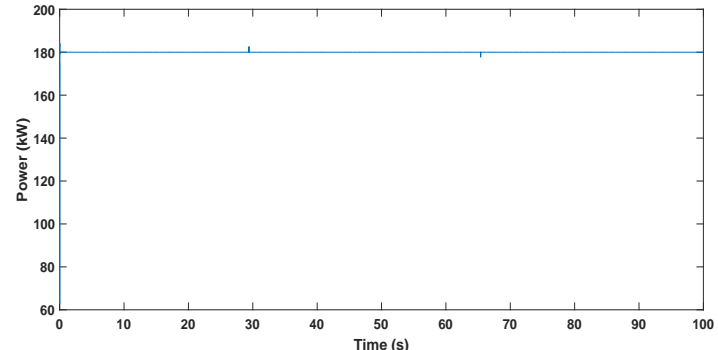

Fig. 11. The total output power of BESSs

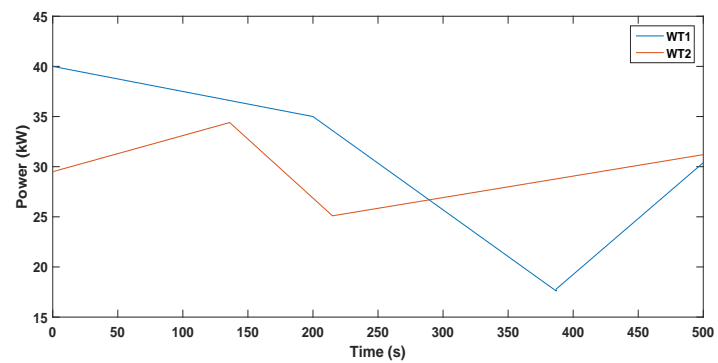

Fig. 12. The output power profile of the wind power generation

of the proposed algorithm. the microgrid is supposed to be islanded in 0s intentionally. The WTs in Fig. 4 are controlled in the reactive power regulation mode, and the output power from each WT is given in Fig. 12, respectively. During the simulation period, each BESS implements the proposed algorithm and is controlled by the strategy in Fig.3. With the coordination scheme in Fig. 2, Figs. 13 - 14 present the results for the output power, the power balance estimation, respectively. It can be observed that the power outputs from BESSs converge to the optimal value, and meanwhile, the supply-demand mismatch is eliminated by the proposed algorithm under the time-varying wind power generations.

\section{Case 5.4}

To extend the proposed algorithm to a large-scale system, the algorithm should be rendered to converging to the optimal value in a timely manner. Thus, the scalability of the proposed algorithm is investigated in this case. To this end, a 30BESS system is built in Matlab/Simulink, and the single line diagram of the 30-BESS system is shown in Fig. 15. The communication network is designed to be weight-balanced and strongly connected, and the supply-demand mismatch

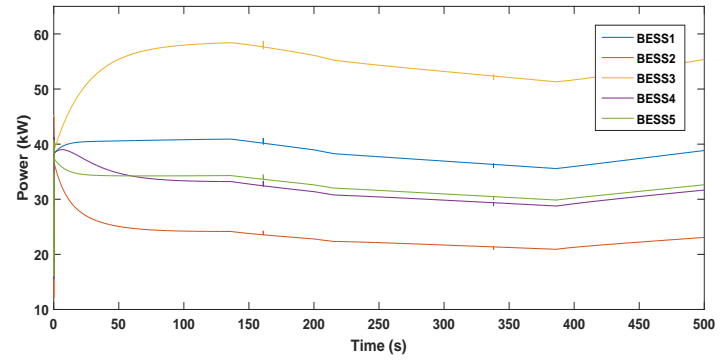

Fig. 13. The output power update under the proposed algorithm 


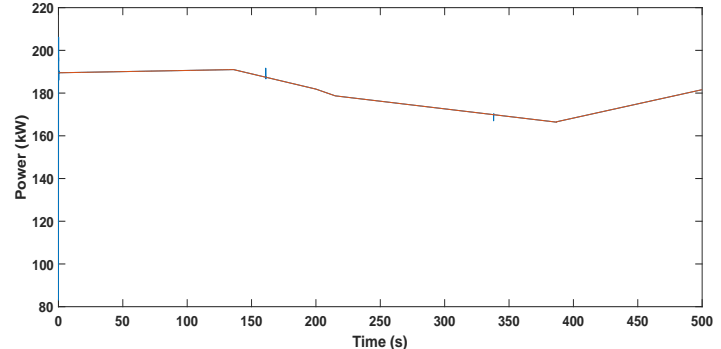

Fig. 14. The power balance estimation

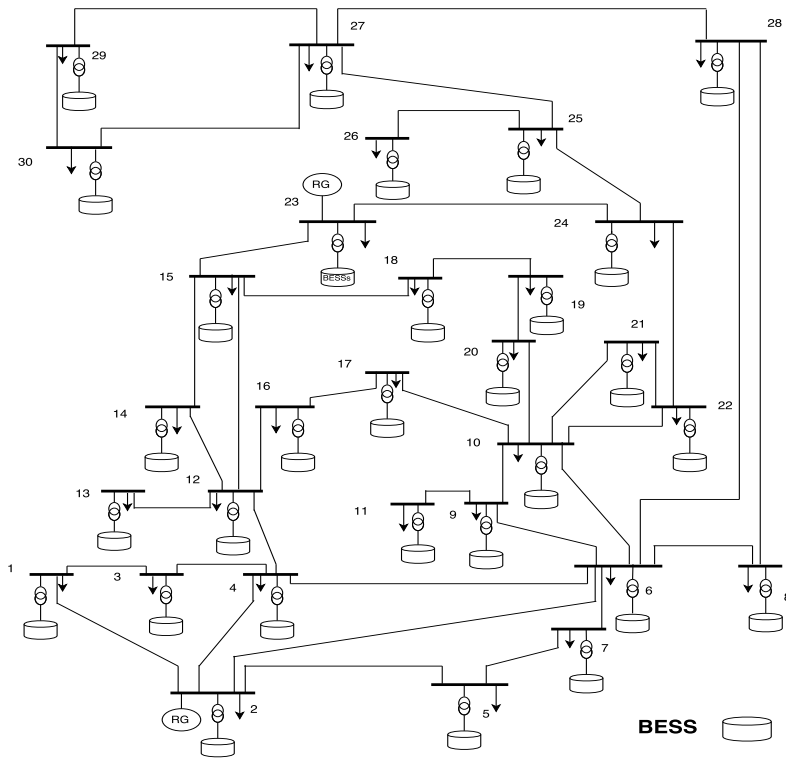

Fig. 15. Single line diagram of the 30-BESS system

is assumed as $1000 \mathrm{~kW}$. The scalability is demonstrated by observing the results in Figs. 16 - 17, which show the output power of each BESS converges to their optimal values while the deviations between demand and supply power converge to zero.

\section{CONCLUSION}

The coordination problem of BESSs in a microgird under high penetration of renewable sources is investigated in this paper. A distributed cooperative control strategy is proposed for BESSs to maintain the supply-demand balance in a microgrid while increasing their profits and energy efficiency. Based on the proposed MAS framework, the proposed solution

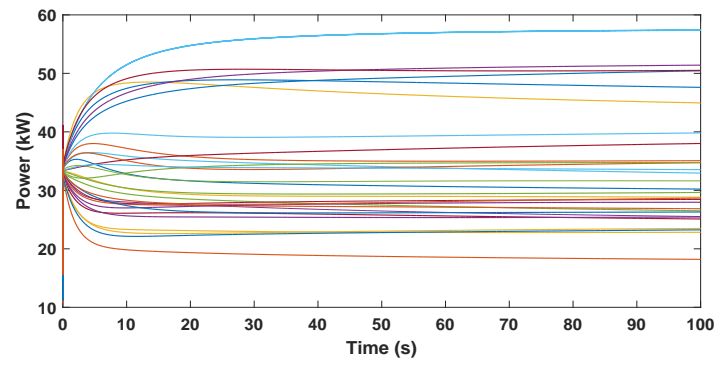

Fig. 16. The output power update under the proposed algorithm

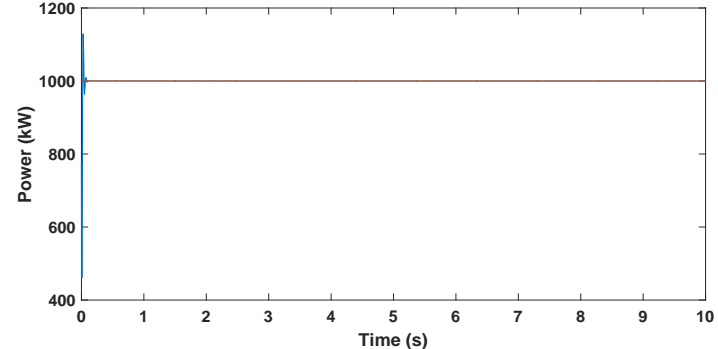

Fig. 17. The power balance estimation

can be implemented in a distributed manner without a central coordinator. Besides, the results indicate that the optimal solution is achieved without releasing the information of the cost function. The effectiveness and the scalability of the proposed distributed strategy are further demonstrated by the simulation using the IEEE test systems and 30-BESS system.

\section{REFERENCES}

[1] F. Katiraei, R. Iravani, N. Hatziargyriou, and A. Dimeas, "Microgrids management," IEEE power energy mag., vol. 6, no. 3, 2008.

[2] F. Wilches-Bernal, J. H. Chow, and J. J. Sanchez-Gasca, "A fundamental study of applying wind turbines for power system frequency control," IEEE Trans. Power Syst., vol. 31, no. 2, pp. 1496-1505, 2016.

[3] J. Aho, A. Buckspan, J. Laks, P. Fleming, Y. Jeong, F. Dunne, M. Churchfield, L. Pao, and K. Johnson, "A tutorial of wind turbine control for supporting grid frequency through active power control," in American Control Conference (ACC), 2012. IEEE, 2012, pp. 31203131.

[4] Y. Wang, G. Delille, H. Bayem, X. Guillaud, and B. Francois, "High wind power penetration in isolated power systems - assessment of wind inertial and primary frequency responses," IEEE Trans. Power Syst., vol. 28, no. 3, pp. 2412-2420, 2013.

[5] L.-R. Chang-Chien, C.-C. Sun, and Y.-J. Yeh, "Modeling of wind farm participation in agc," IEEE Trans. Power Syst., vol. 29, no. 3, pp. 12041211, 2014.

[6] S. Aditya and D. Das, "Application of battery energy storage system to load frequency control of an isolated power system," International journal of energy research, vol. 23, no. 3, pp. 247-258, 1999.

[7] X. Li, D. Hui, and X. Lai, "Battery energy storage station (bess)based smoothing control of photovoltaic (pv) and wind power generation fluctuations," IEEE Trans. Sustain. Energy, vol. 4, no. 2, pp. 464-473, 2013.

[8] G. He, Q. Chen, C. Kang, Q. Xia, and K. Poolla, "Cooperation of wind power and battery storage to provide frequency regulation in power markets," IEEE Transactions on Power Systems, 2016.

[9] A. G. Tsikalakis and N. D. Hatziargyriou, "Centralized control for optimizing microgrids operation," IEEE Trans. Energy Convers., vol. 23, no. 1, pp. 241-248, 2008.

[10] K. Tan, X. Peng, P. L. So, Y. C. Chu, and M. Chen, "Centralized control for parallel operation of distributed generation inverters in microgrids," IEEE Trans. Smart Grid, vol. 3, no. 4, pp. 1977-1987, 2012.

[11] H. Xin, Z. Qu, J. Seuss, and A. Maknouninejad, "A self-organizing strategy for power flow control of photovoltaic generators in a distribution network," IEEE Trans. Power Syst., vol. 26, no. 3, pp. 1462-1473, 2011.

[12] G. Mokhtari, G. Nourbakhsh, and A. Ghosh, "Smart coordination of energy storage units (esus) for voltage and loading management in distribution networks," IEEE Trans. Power Syst., vol. 28, no. 4, pp. 4812-4820, 2013.

[13] Z. Wang, K. Yang, and X. Wang, "Privacy-preserving energy scheduling in microgrid systems," IEEE Trans. Smart Grid, vol. 4, no. 4, pp. 18101820, 2013.

[14] P. Mercier, R. Cherkaoui, and A. Oudalov, "Optimizing a battery energy storage system for frequency control application in an isolated power system," IEEE Trans. Power Syst., vol. 24, no. 3, pp. 1469-1477, 2009.

[15] S.-J. Lee, J.-H. Kim, C.-H. Kim, S.-K. Kim, E.-S. Kim, D.-U. Kim, K. K. Mehmood, and S. U. Khan, "Coordinated control algorithm for distributed battery energy storage systems for mitigating voltage and 
frequency deviations," IEEE Transactions on Smart Grid, vol. 7, no. 3, pp. 1713-1722, 2016.

[16] T. R. Oliveira, W. W. A. G. Silva, and P. F. Donoso-Garcia, "Distributed secondary level control for energy storage management in dc microgrids," IEEE Transactions on Smart Grid, 2016.

[17] R. Wang, H. Tang, and Y. Xu, "Distributed cooperative optimal control of energy storage systems in a microgrid," in 2016 IEEE Power and Energy Society General Meeting (PESGM), July 2016, pp. 1-5.

[18] K. Tsang, L. Sun, and W. Chan, "Identification and modelling of lithium ion battery," Energy Conversion and Management, vol. 51, no. 12, pp. 2857-2862, 2010.

[19] J. Choi, I. Choi, G. An, and D. J. Won, "Advanced power sharing method for energy efficiency improvement of multiple battery energy storages system," IEEE Transactions on Smart Grid, vol. PP, no. 99, pp. 1-1, 2016.

[20] Y. Xu, W. Zhang, W. Liu, X. Wang, F. Ferrese, C. Zang, and H. Yu, "Distributed subgradient-based coordination of multiple renewable generators in a microgrid," IEEE Trans. Power Syst., vol. 29, no. 1, pp. 23-33, 2014.

[21] P. Samadi, A.-H. Mohsenian-Rad, R. Schober, V. W. Wong, and J. Jatskevich, "Optimal real-time pricing algorithm based on utility maximization for smart grid," in Smart Grid Communications (SmartGridComm), 2010 First IEEE International Conference on. IEEE, 2010, pp. 415-420.

[22] A.-H. Mohsenian-Rad and A. Leon-Garcia, "Optimal residential load control with price prediction in real-time electricity pricing environments," IEEE trans. Smart Grid, vol. 1, no. 2, pp. 120-133, 2010.

[23] C. Zhao, J. He, P. Cheng, and J. Chen, "Consensus-based energy management in smart grid with transmission losses and directed communication," IEEE Transactions on Smart Grid, 2016.

[24] L. Wong, "A review of transmission losses in planning studies," California Energy Commission, 2011.

[25] H. H. Moon, J. J. Lee, S. Y. Choi, J. S. Cha, J. M. Kang, J. T. Kim, and M. C. Shin, "A study using a monte carlo method of the optimal configuration of a distribution network in terms of power loss sensing," Sensors, vol. 11, no. 8, pp. 7823-7834, 2011.

[26] N. Rahbari-Asr, U. Ojha, Z. Zhang, and M.-Y. Chow, "Incremental welfare consensus algorithm for cooperative distributed generation/demand response in smart grid," IEEE Trans. Smart Grid, vol. 5, no. 6, pp. 2836-2845, 2014.

[27] F. Chen, M. Chen, Q. Li, K. Meng, Y. Zheng, J. Guerrero, and D. Abbott, "Cost based droop schemes for economic dispatch in islanded microgrids," 2016.

[28] S. Boyd, N. Parikh, E. Chu, B. Peleato, and J. Eckstein, "Distributed optimization and statistical learning via the alternating direction method of multipliers," Foundations and Trends $₫$ in Machine Learning, vol. 3, no. 1, pp. 1-122, 2011.

[29] M. Avriel, Nonlinear programming: analysis and methods. Courier Corporation, 2003.

[30] M. Tahir and S. K. Mazumder, "Self-triggered communication enabled control of distributed generation in microgrids," IEEE Trans, Ind. Informat., vol. 11, no. 2, pp. 441-449, 2015.

[31] K. J. Arrow, L. Hurwicz, and H. Uzawa, Studies in linear and non-linear programming, ser. Stanford Math. Stud. Social Sci. Stanford, CA: Cambridge Univ. Press, 1958. [Online]. Available: https://cds.cern.ch/record/222917

[32] S. S. Kia, "A distributed dynamical solver for an optimal resource allocation problem over networked systems," in Decision and Control (CDC), 2015 IEEE 54th Annual Conference on. IEEE, 2015, pp. 74827487.

[33] R. Wang, H. Tang, and Y. Xu, "Distributed cooperative optimal control of energy storage systems in a microgrid," in Power and Energy Society General Meeting (PESGM), 2016. IEEE, 2016, pp. 1-5.

[34] Z. Fu, X. He, T. Huang, and H. Abu-Rub, "A distributed continuous time consensus algorithm for maximize social welfare in micro grid," Journal of the Franklin Institute, vol. 353, no. 15, pp. 3966-3984, 2016.

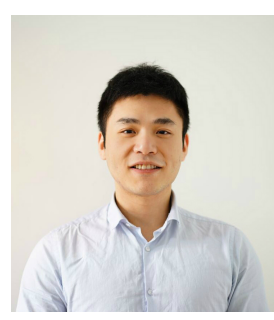

Tianqiao Zhao received his B.Eng. degree in automatic control from North China Electric Power University, Hebei, China, in 2013, and his M.Sc degree in electrical and electronic engineering from the University of Manchester, U.K., in 2014. He is now a Ph.D. candidate in control engineering with the School of Electrical and Electronic Engineering at the University of Manchester, U.K. His research interests include distributed optimization of microgrids, distributed control and energy storage systems.

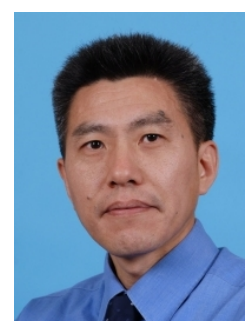

Zhengtao Ding received the B.Eng. degree from Tsinghua University, Beijing, China, and the M.Sc. degree in systems and control and the Ph.D. degree in control systems from the University of Manchester Institute of Science and Technology, Manchester, U.K. After working as a Lecturer with Ngee Ann Polytechnic, Singapore, for ten years, in 2003, he joined The University of Manchester, Manchester, U.K., where he is currently a Senior Lecturer of control engineering with the School of Electrical and Electronic Engineering. He is the author of the book Nonlinear and Adaptive Control Systems (IET, 2013) and a number of journal papers. His research interests include nonlinear and adaptive control theory and their applications. Dr. Ding serves as an Associate Editor for the IEEE Transactions on AUtOMATIC CONTROL, Transactions of the Institute of Measurement and Control, Control Theory and Technology, Mathematical Problems in Engineering, Unmanned Systems, and the International Journal of Automation and Computing. 